\title{
Artificial Smart Composites: Bionics Principles
}

\author{
Serge Shilko* \\ V.A. Belyi Metal-Polymer Research Institute of NANB, Belarus, Russia
}

*Corresponding author: Serge Shilko, V.A. Belyi Metal-Polymer Research Institute of NANB, Belarus, Russia.

Received Date: May 21, 2020

Published Date: June 09, 2020

\section{Opinion}

The search for effective ways to achieve the synergy of the matrix and structurally active (reinforcing, antifriction, etc.) dispersed and spatially oriented fibrous fillers is a general trend in the development of composite materials science. Despite the significant amount of experimental data available and the variety of matrix materials, modifiers and technologies, it is relevant to develop new approaches to creating structural and functional composites with rapid prototyping and testing of products based on them.

Along with lately requisite properties such as stability of physical and mechanical characteristics, linearity of the equation of state and unambiguity of response to disturbance, there arose a problem of a complex active response to varying outer conditions. Other words, a tendency is observed of increasing number of material functions acquiring the features of intellectual systems [1].

New opportunities are associated with the development of an interdisciplinary concept that provides for the joint use of the achievements of the physics and mechanics of solids, multiscale modeling of structurally heterogeneous materials [2,3], as well as bionics, nano-, additive and new information technologies (conception of digital twins).

In particular, research in the field of cybernetics [4], principles of self-organization [5] and bionics developing ideas about the basic laws of life, contributes to innovation in all technical fields, without exception, from prosthetics to global control and production systems.

Living systems optimized evolutionarily have been and remain a source of effective design, technological and material science solutions. Biomaterials are characterized by appropriate adaptive reactions to changes in external conditions, which contributes to the achievement of extremely valuable stress-strain, tribological and other indicators (equal strength, lack of gravity, self-healing, damping, ultra-low friction, etc.).

So, obvious prototypes of these materials turn to be biosystems, from the one hand, and computer monitored technical systems able to reproduce intellectual behavior using sensor, processor and executive functions (including effector function and response action), from the other hand, plus feedforward and feedback. Although means of these properties' realization can't be similar in artificial materials and above-mentioned natural prototypes, generalizations obtained at the junction of the materials science, bionics and cybernetics allow to formulate the conceptual principles and to consider probable ways of the named interdisciplinary problem solution.

Recent reviews and terminological discussions in the field have confirmed actuality of the structural and functional analyses of smart composites, including functional nanomaterials. However, papers, devoted to such materials are commonly reduced to creation of sensors and actuators. Less attention has received principles and models of intellectual (processor) reactions in artificial materials. The development of adaptive composites allows us to hamper the failure process and promotes reliability and service life of products for different technical applications.

It is fundamentally important to describe the heterogeneity and mobility of the interphase boundary in bionically adequate materials, take into account the feedback between the diagnosed and output parameters, and analyze the processes of structure self-organization in real time. To this end, micromechanical representations, variational methods for solving problems on 
a moving boundary, regularization methods for solving inverse problems, etc., as well as experimental methods for studying the structure and local mechanical properties (atomic force microscopy, tomography) are involved.

The proposed opinion in structural design of bionically adequate composites under development includes:

- taxonomy [6] of materials based on a multidimensional structural and functional analysis and the theory of synergetic;

- development of micromechanical models of materials (porous, granular, polymers, reinforced composites, biological tissues) with the allocation of a representative volume and structural units of the deformable medium;

- the formulation of the physical mechanisms of functioning of smart materials, as thermodynamically open systems with metastable phase composition and feedbacks, in which

Table 1: Evolution of structure and properties of materials.

\begin{tabular}{|c|c|c|c|}
\hline Generation of materials & Structural-and-functional characteristics & Means of property regulation & Factor determining optimum result \\
\hline Traditional material & $\begin{array}{c}\text { Monofunctional single-component ma- } \\
\text { terial }\end{array}$ & $\begin{array}{c}\text { Properties determined a priori by the } \\
\text { origin of component material }\end{array}$ & Initial property of monocomponent \\
\hline $\begin{array}{c}\text { Composite material } \\
\text { material with fixed boundaries between } \\
\text { components }\end{array}$ & $\begin{array}{c}\text { Properties are efficiently regulated } \\
\text { technologically based on principles of } \\
\text { adaptation and synergism }\end{array}$ & $\begin{array}{c}\text { Initial property of components and } \\
\text { interlayers }\end{array}$ \\
\hline $\begin{array}{c}\text { Smart (adaptive) com- } \\
\text { posites }\end{array}$ & $\begin{array}{c}\text { Polyfunctional polycomponental material } \\
\text { with movable boundaries between com- } \\
\text { ponents }\end{array}$ & $\begin{array}{c}\text { Self-regulation of structure based on } \\
\text { sensor, processor and effector functions } \\
\text { and feedforward and feedback channels }\end{array}$ & $\begin{array}{c}\text { Efficiency of sensing extreme ef- } \\
\text { fects and elimination of refusals }\end{array}$ \\
\hline
\end{tabular}

The first generation is traditional materials including monofunctional media whose properties are determined by the nature and initial quality of a single component. The next are traditional composites with a prominent structural hierarchy, being also monofunctional. They are characterized by stability of inner and external boundaries, i.e. fixed structure of components, intermediate layers and the composite as a whole.

Adaptive materials with coordinated functions and active behavior belong to the third advanced generation of materials. These systems perceive outer effects at unchanged function owing to, presumably, structural self-organization. In this connection, the mobility of the component boundaries should be remembered as automatic expedient restructuring of the structure is realized under extreme external influence in accordance with a given criterion of optimality;

- mathematical justification of the ability to control the deformation, friction, and fracture modes of artificial composite materials to implement their smart (quasi-biological) behavior, in particular, based on solutions to the problems of localizing the moving interfaces, including determining the optimal gradient of the interfacial layers.

Some words about classification of materials with account of interrelations found between structure and functions as well as analysis and modeling of a subclass of intellectual systems, namely adaptive composite materials. These assumptions are based on the theory of functional systems and synergism [5]. Three generations of materials which can be discriminated in the proposed classification, are given in Table 1.

an indispensable property of smart materials, which is not present in traditional composites.

The qualitative transition of materials from the passive to active functioning is shown in Table 2. Naturally, prerequisites of such a transition are formed at the levels of two preceding generations. Thus, transformation of one physical field into another (e.g., piezo- or photo effects) is probable at the stage of monofunctional material. The creation of qualitatively new (emerged), including forecast properties, is a logical continuation of the additive and synergetic principles of composite production. This precedes the development of adaptive composites, being a subclass of smart systems with the dominating adaptive strategy.

Table 2: Systematization of materials by general criteria.

\begin{tabular}{|c|c|c|c|c|}
\hline Functional evolution & Degree of activity & Degree of intellect & Functioning quality & Mode of behavior \\
\hline \multirow{2}{*}{ mono-functional } & passive & "trivial" & \multirow{2}{*}{ material } & \multirow{2}{*}{ "forecast" } \\
\hline & active & “wit" (functional) & & \\
\hline \multirow{7}{*}{ poly-functional } & \multirow{7}{*}{ active } & \multirow{3}{*}{ smart (adaptive) } & \multirow{2}{*}{ material $=$ part } & "indefinite" \\
\hline & & & & "egoist" \\
\hline & & & material = system & "time-server" \\
\hline & & \multirow{4}{*}{ "wise" (ecophilous) } & \multirow{4}{*}{ material $=$ medium } & "kamikaze" \\
\hline & & & & "regenerate" \\
\hline & & & & "cyber" \\
\hline & & & & "incognito" \\
\hline
\end{tabular}


The suggested classification makes it possible to forecast other unknown materials of the intellectual type, for example, capable of self-destruction "kamikaze", those ensuring partial or full restoration "regenerators" and materials offering programmed control of the environment ("cyber") and implicit ("incognito") ones. These subclasses constitute a new type of "ecophilous" materials which behavior supports homeostasis of the environment.

Relative simplicity of adaptive composite materials is due to their orientation aimed to fulfill only the adaptive function of the part or a system in contrast to a higher status of the materialmedium subclass (Table 2). However the adaptive composite is formed rather in time than by a mechanical mixing of structural components, and revolutionizes as a specific unit by coordinating interrelated physical processes based on an imparted optimum criterion. In this case, the emergence of macrostructure is specified by origination of collective modes under the action of fluctuations, there competing and, finally, by selection of the most accommodated mode or their combination. The structures themselves could be described in physical terms as types of adaptation to outer conditions.

Reaction of a material due to mutual coordination of structural and functional parameters of microsystems characterizes it as an open self-regulating system. Selection of the mode of behavior in response to outer effect does not arise from the principle of the least action, neither from the principle of compulsion (Gauss principle) nor from that of the utmost probability. Active response systems eliminate (or subordinate) contingency. This makes grounds to speak about a programmed behavior of the system, i.e. the decision is made according to the inner criteria determined by the structure itself and system parameters, which substantiates the necessity of direct and reverse connection channels.

It follows from the above said that to form a more complex processor function of smart materials it is possible to use the universe phenomenon of self-organization, which is not limited to only systems of higher organization and functional complexity and isn't a monopoly of bio- or social systems. A self-organizing system is understood as a system capable of stabilizing parameters under varying outer conditions through directed ordering of its structural and functional relations aimed at withstanding entropic factors of the environment, which helps to preserve its characteristics as an integral formation [5].

The material formed by combining its components acquires the characteristics of a composite structure, which is a notion nonequivalent to the structure of its constituents. This fact raises composite materials to a higher structural level and admits the probability of per layer differentiation of the functions in order to reach the integral control system. In our view, to realize adaptation mechanism to outer conditions in composite materials, it's worthwhile considering the combination of different scale physical processes, where we single out at least 4 structural levels: molecular, mesoscopic, macroscopic and polycomponental (Figure 1).

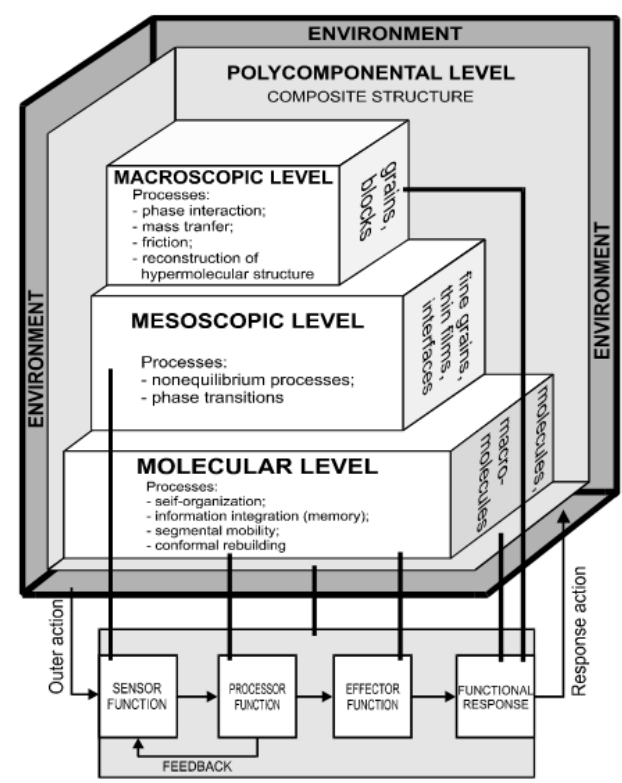

Figure 1: Differentiation of structural levels as a cornerstone of material science.

The molecular level is the basic one at programming material behavior. This is because its scale in polymer composites corresponds to cooperative effects of segmental mobility and conformal rebuilding that provide conditions for self-organization in high-molecular bodies. Just here the processor function is realized as a capacity for estimating variations due to outer effects and as a tool formulating the character and force of response based on stationary characteristics of the microsystem. Also, the effector 
function is fulfilled here for exciting reverse reactions by varying characteristics of the microsystem on a self-organization base.

The mesoscopic level performs the sensor function as an ability to perceive outer effects. Non-equilibrium processes are initiated at this level changing molecular structure and supporting the interaction of direct and reverse channels between the levels.

The macroscopic level makes provision for the mobile function as a reorganization of the initial subsystems (components) aimed at preserving the behavior model.

The mobile function is also realized at the polycomponental level, though intention in this case to provide the system (material $=$ article) functioning as a whole.

To organize control, the processes relating to the mention levels should be coordinated using functional links between them.

It is to be remembered that polymer composites are potential carriers of intellectual properties. Namely, they are sensitive to physical fields, i.e. show a sensor function; make it possible to carry out the actuator function (shape memory of thermosetting resins, etc) and, finally, among all other artificial material media they most closely approach the living nature (bio tissues are usually built of high-molecular compounds).

The study of synergetic phenomena in nonliving nature as a linking element between analogous processes in original objects will, in our opinion, provide a possibility to find structural-andfunctional bio prototypes of smart composites.

\section{Conclusion}

- The systematic analysis of the problem of developing bionically adequate smart composites has enabled us to trace evolution of structural organization of artificial materials, to clarify the mechanisms of adaptation to the external action, and to disclose, to a certain degree, the effect of structure on formation of the optimum back reaction.

- It seemed that mathematical description of smart composite structures is formulated as a problem on localizing moving interfaces. The study of synergetic phenomena in the nonliving nature and analogous processes in biological objects will, in our opinion, provide a possibility to find structural-andfunctional prototypes of adaptive composites.

- Time will tell, to what extent the proposed approaches may prove to be fruitful and useful for the materials science and the material production as a whole.

\section{Acknowledgment}

This work was supported by the Belarusian Republican Foundation for Fundamental Research under project T20R-223 (No. 18-58-00037). The author is grateful to Prof. Yu. Pleskachevsky for helpful discussion.

\section{Conflict of Interest}

No conflict of interest

\section{References}

1. Wang ZL (1998) Functional and Smart materials: Structural Evolution and Structure Analysis. Kluwer Academic Publishers, Boston.

2. Lazzari B, M Fabrizio, A Morro (2002) Mathematical Models and Methods for Smart Materials. World Sci Publ, New Jersey.

3. Laso M (2009) Smart Materials Modelling. J Wiley \& Sons, NY.

4. Wiener N (1948) Cybernetics or Control and Communication in the Animal and the Machine. MIT Press, Cambridge.

5. Prigogine I, Stengers I (1984) Order out of Chaos: Man's new dialogue with nature, Flamingo, ISBN 0006541151, London.

6. Goldade V, Shilko S, Neverov A (2015) Smart Materials Taxonomy. CRC Press, Taylor \& Francis Group, pp. 277. 